\title{
Front Matter: Volume 11345
}

, "Front Matter: Volume 11345," Proc. SPIE 11345, Nanophotonics VIII, 1134501 (12 May 2020); doi: 10.1117/12.2571814

SPIE. Event: SPIE Photonics Europe, 2020, Online Only 


\title{
PROCEEDINGS OF SPIE
}

\section{Nanophotonics VIII}

\author{
David L. Andrews \\ Angus J. Bain \\ Martti Kauranen \\ Jean-Michel Nunzi \\ Editors
}

\section{6-10 April 2020 \\ Online Only, France}

Sponsored by

SPIE

Cosponsored by

City of Strasbourg (France)

Eurometropole (France)

CNRS (France)

Région Grand Est (France)

iCube (France)

Université de Strasbourg (France)

Cooperating Organisations

Photonics 21 (Germany)

EOS-European Optical Society (Germany)

Photonics Public Private Partnership (Belgium)

Photonics France (France)

Published by

SPIE 
The papers in this volume were part of the technical conference cited on the cover and title page. Papers were selected and subject to review by the editors and conference program committee. Some conference presentations may not be available for publication. Additional papers and presentation recordings may be available online in the SPIE Digital Library at SPIEDigitalLibrary.org.

The papers reflect the work and thoughts of the authors and are published herein as submitted. The publisher is not responsible for the validity of the information or for any outcomes resulting from reliance thereon.

Please use the following format to cite material from these proceedings:

Author(s), "Title of Paper," in Nanophotonics VIII, edited by David L. Andrews, Angus J. Bain, Martti Kauranen, Jean-Michel Nunzi, Proceedings of SPIE Vol. 11345 (SPIE, Bellingham, WA, 2020) Sevendigit Article CID Number.

ISSN: 0277-786X

ISSN: 1996-756X (electronic)

ISBN: 9781510634626

ISBN: 9781510634633 (electronic)

Published by

SPIE

P.O. Box 10, Bellingham, Washington 98227-0010 USA

Telephone +1360 6763290 (Pacific Time) · Fax +1 3606471445

SPIE.org

Copyright (c) 2020, Society of Photo-Optical Instrumentation Engineers.

Copying of material in this book for internal or personal use, or for the internal or personal use of specific clients, beyond the fair use provisions granted by the U.S. Copyright Law is authorized by SPIE subject to payment of copying fees. The Transactional Reporting Service base fee for this volume is $\$ 21.00$ per article (or portion thereof), which should be paid directly to the Copyright Clearance Center (CCC), 222 Rosewood Drive, Danvers, MA 01923. Payment may also be made electronically through CCC Online at copyright.com. Other copying for republication, resale, advertising or promotion, or any form of systematic or multiple reproduction of any material in this book is prohibited except with permission in writing from the publisher. The CCC fee code is $0277-$ $786 \mathrm{X} / 20 / \$ 21.00$.

Printed in the United States of America by Curran Associates, Inc., under license from SPIE.

Publication of record for individual papers is online in the SPIE Digital Library.

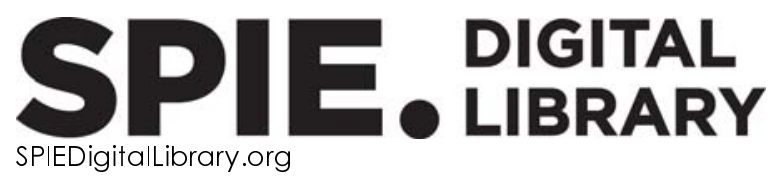

Paper Numbering: Proceedings of SPIE follow an e-First publication model. A unique citation identifier (CID) number is assigned to each article at the time of publication. Utilization of CIDs allows articles to be fully citable as soon as they are published online, and connects the same identifier to all online and print versions of the publication. SPIE uses a seven-digit CID article numbering system structured as follows:

- The first five digits correspond to the SPIE volume number.

- The last two digits indicate publication order within the volume using a Base 36 numbering system employing both numerals and letters. These two-number sets start with 00, 01, 02, 03, 04, 05, 06, 07, 08, 09, OA, OB ... 0Z, followed by 10-1Z, 20-2Z, etc. The CID Number appears on each page of the manuscript. 


\section{Contents}

$\begin{array}{ll}\text { vii } & \text { Authors } \\ \text { ix } & \text { Conference Committee }\end{array}$

STRUCTURED LIGHT AND APPLICATIONS

1134507 Probing the backscattering of $\mathrm{TiO}_{2}$ particles with vortex beams [1 1345-6]

OPTICAL MANIPULATION

$113450 G$ Passive laser irradiation as a tool for optical catalysis [11345-15]

PLASMONICS I

$11345 \mathrm{OM}$ Engineering the emission statistics of plasmonic lasers [11345-22]

1134500 Metals and dielectrics for UV plasmonics [1 1345-24]

PLASMONICS AND NANOANTENNAE

$113450 X \quad$ Evanescent and propagating fields of a strongly focused beam in the near-field and far-field regions [11345-34]

11345 OY Study of absorption and emission dipoles of upconverting nanoparticles optically trapped at the absorption resonance [11345-35]

NOVEL SYNTHETIC METHODS

$1134510 \quad$ Novel supra-molecular arrangements with plasmonic functionalities for fipronil pesticide detection [11345-37]

1134512 Core-shell magneto-luminescent nanocomposites [11345-39] 
SENSING

$1134516 \quad$ Long-wave infrared spectral filter with semiconductor materials [11345-43]

PHOTOINDUCED EFFECTS

11345 1B Analysis of Förster resonance energy transfer (FRET) in the vicinity of a charged metallic nanosphere via nonlocal optical response method [11345-48]

$113451 \mathrm{C}$ Spontaneous emission of atomic dipoles near two-sided semi-transparent mirrors [11345-49]

11345 1D Long-range coupling of individual quantum dots with plasmonic nanoparticles in a thin-film hybrid material [1 1345-50]

$113451 \mathrm{E} \quad$ Photoinduced change in SPIONs/CdSe/ZnS nanocomposites optical properties [11345-51]

PHOTONIC SOURCES

$1134511 \quad$ Mid-infrared radiation source for spectroscopic applications based on multi-walled carbon nanotubes on top of silicon [1 1345-56]

GUIDED MODES AND CAVITIES

$113451 \mathrm{~J} \quad$ Silicon nitride based guided mode resonance structures for enhancement of nonlinear optical effects [11345-58]

$113451 \mathrm{~L} \quad$ Analysis of multipolar contributions to eigenmodes in resonators of various shapes [11345-60]

TIME RESOLVED DYNAMICS

$113451 \mathrm{M}$ Coherent carrier and exciton transport in organic semiconductors (Invited Paper) [11345-61]

NONLINEAR INTERACTIONS

$113451 R$ Second harmonic generation from an ITO nanolayer: experiment versus theory [1 1345-66]

11345 1S Absolute two-photon absorption cross-sections of single-exciton states in semiconductor nanocrystals [11345-67]

iv 
$113451 \mathrm{~T} \quad$ Three-dimensional nonlinear plasmonic metamaterials [11345-68]

POSTER SESSION

$1134521 \quad$ Surface plasmon polariton generation in a carbon nanotube on dielectric substrate [1 1345-25]

1134522 SERS spectroscopy and quantum-chemical calculations of stilbene amino derivatives in conditions of adsorption on silver nanoparticles [11345-70]

1134523 Enhanced spontaneous emission in Anderson localized cavities [1 1345-71]

1134524 Generalized Brewster effect in aluminum-doped ZnO nanopillars [1 1345-72]

1134527 Targeted genome editing in potato protoplast via optical delivery of CRISPR/Cas9 ribonucleoproteins [11345-76]

1134529 Classical electromagnetism at thermal equilibrium [11345-80]

11345 2A Performance comparison of tin-based group IV SQWIP and MQWIP in dark conditions [11345-81]

$113452 \mathrm{~B} \quad$ Optical parameters of coupled vertical cylindrical quantum dots with double modified Pöschl-Teller potential in terahertz range [1 1345-82]

$113452 \mathrm{C} \quad$ Sensing solutions for SERS applications using gold nanoparticle modified quartz surfaces [1 1345-83]

$113452 F \quad$ Multifunctional, flexible, and free-standing SERS-active AgNW filter foils [1 1345-86]

$1134521 \quad$ New method of SERS-active gold surfaces fabrication for bacterial cells Raman analysis [1 1345-89]

$113452 \mathrm{~J} \quad$ Dielectric nanocavity for the emission control of a single-photon source [1 1345-90]

$113452 \mathrm{~K}$ Investigation of GeSn/SiGeSn nanostructured layer for sensors in mid-infrared application [1 1345-91]

$113452 \mathrm{~L} \quad$ FTDT numerical calculatons of local plasmonic fields for multilayer gold nanoparticles-agents for theranostics [1 1345-92]

$113452 \mathrm{M} \quad$ Polarization-independent SERS substrates for trace detection of chemical and biological molecules [1 1345-94]

1134520 Optical properties of hybrid film depending on overlap of the nanoparticle plasmon resonance and J-aggregate band of cyanine dye [1 1345-96]

$113452 \mathrm{P} \quad$ Laser fabrication of hybrid nanostructures with nonlinear response [1 1345-97] 
$113452 \mathrm{~V} \quad$ Investigation of InAs quantum dots grown on the Ge substrate without migration enhance epitaxy layer [1 1345-105]

11345 2W Influence of InGaAs matrix thickness on the optical properties and strain distribution in selfassembled sub-monolayer InAs quantum dot heterostructures [11345-106]

$113452 X \quad$ Study on optical properties and strain distribution of InAs/InGaAs sub-monolayer quantum dot heterostructure with multiple stacking layers [11345-107]

$113452 Y \quad$ Effect of varying facet angle on the strain and energy band profile of InAs QD heterostructures [11345-108]

1134522 A comparative analysis of analog and digital alloy technique of $\ln _{\mathrm{x}} \mathrm{Ga}_{1-\mathrm{x}}$ As capping material on InAs quantum dot heterostructures [11345-109] 


\section{Authors}

Numbers in the index correspond to the last two digits of the seven-digit citation identifier (CID) article numbering system used in Proceedings of SPIE. The first five digits reflect the volume number. Base 36 numbering is employed for the last two digits and indicates the order of articles within the volume. Numbers start with 00, 01, 02, 03, 04, 05, 06, 07, 08, 09, 0A, 0B...0Z, followed by 10-1Z, 20-2Z, etc.

\author{
Abeywickrama, Champi Asoka, 1B \\ Ageev, Eduard, 2J, 2P \\ Akdemir, Ozan, $2 \mathrm{~F}$ \\ Akozbek, N., 1R \\ Albella, P., 00 \\ Alcaraz de la Osa, R., 00 \\ Andrews, David L., OG, 1B \\ Baranov, M. A., 12 \\ Bartels, Frederik-Matti, 27 \\ Beige, Almut, 1C \\ Bek, Alpan, 2F \\ Bentivegna, Florian F. L., 21 \\ Binder, Robert, $1 M$ \\ Biswas, Rabindra, $1 \mathrm{~J}$ \\ Boch, Jens, 27 \\ Bogdanov, Andrey, $1 \mathrm{~L}$ \\ Bourouina, Tarik, 11 \\ Brey, Dominik, $1 \mathrm{M}$ \\ Burghardt, Irene, $1 \mathrm{M}$ \\ Castagna, R., 10 \\ Cerutti, Laurent, 16 \\ Chakrabarti, Subhananda, 2V, 2W, 2X, 2Y, $2 Z$ \\ Chatterjee, Sharmistha, 24 \\ Cojocaru, C., IR \\ Coppens, Z. J., $1 R$ \\ Coşkun, Şahin, 2F \\ Czaplicki, Robert, $1 \mathrm{~T}$ \\ Dadoenkova, Yuliya, 21 \\ Das, A., $2 \mathrm{M}$ \\ Dawson, Benjamin, 1C \\ Demirtas, Özge, 2F \\ Demishkevich, Elizaveta A., 2C \\ Dhawan, A., 2M \\ Dongre, Suryansh, $2 \mathrm{~V}$ \\ Dubavik, Alekey U., 12, 1E \\ Dyagileva, Daria V., 1D, 1S \\ Elsayed, Ahmed A., 11 \\ Everitt, H. O., $0 \mathrm{O}$ \\ Forbes, Kayn A., OG \\ Frizyuk, Kristina, 1L \\ Furtak-Wells, Nicholas, $1 \mathrm{C}$ \\ Gazi, Sanowar Alam, 2V, 2W, 2X \\ Genevet, Patrice, $1 \mathrm{~T}$ \\ Gladskikh, Igor A., 20 \\ Gladyshev, Sergey, $1 \mathrm{~L}$ \\ Gökbulut, Belkis, 23 \\ González, F., 00 \\ González-Posada Flores, Fernando, 16 \\ Grachkova, Ekaterina, 2P
}

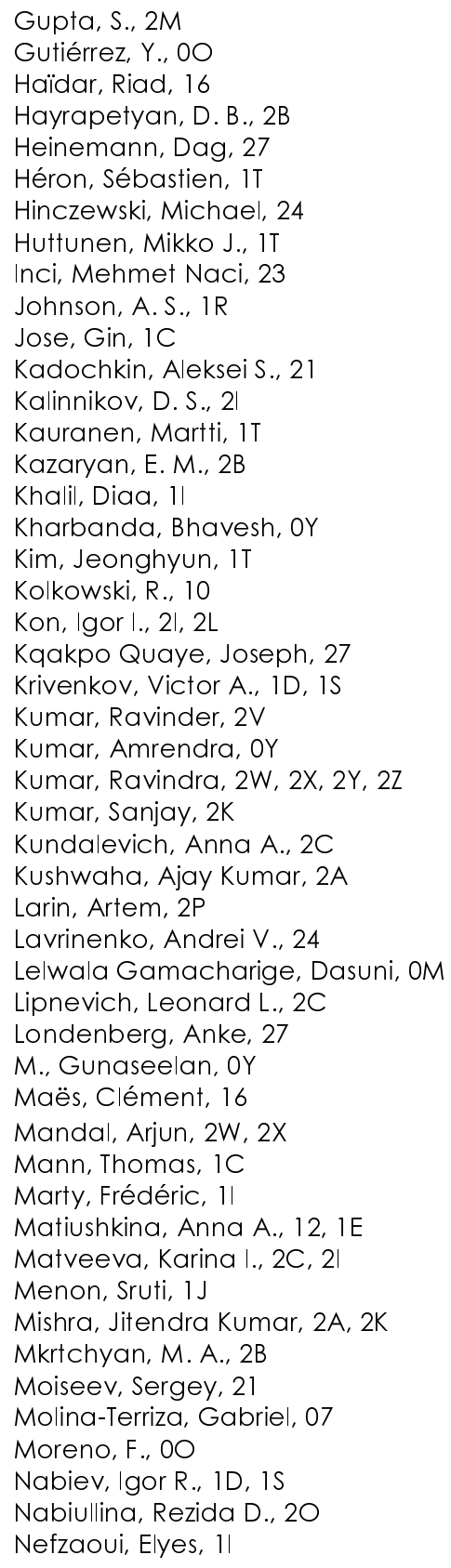


Odintsova, Olga $\vee ., 22$

Orlova, Anna O., 12, $1 \mathrm{E}$

Ortiz, D., 00

Ove, Daigo, 29

Panda, Debiprasad, 2V, 2W, 2X

Pandey, Saurabh Kumar, 2A, 2K

Pareek, Prakash, 2A, 2K

Perez-Salinas, D., $1 \mathrm{R}$

Petrov, Nikolai I., OX

Petti, L., 10

Popp, Wjatscheslaw, 1M

Premaratne, Malin, OM, 1B

Prosad, Asish, $1 \mathrm{~J}$

Raghunathan, Varun, $1 \mathrm{~J}$

Rakovich, Yury P., 1D

Ranjan, Ravi, 2A, 2K

Reddy Shriram, Saranya, 2W, 2X

Reznik, Ivan A., 1E

Rho, Junsuk, IT

Ripken, Tammo, 27

Rippa, M., 10

Rodriguez-Suné, L., IR

Roy, Basudev, OY

Sabry, Yasser M., 11

Saeed, Ahmed, 11

Saha, Jhuma, 2V, 2W, 2X, 2Y, $2 Z$

Saiz, J. M., 00

Samokhvalov, Pavel S., 1D, is

Samusev, llia G., 2C, 2l, 2 L

Sarkisyan, H. A., 2B

Scalora, M., IR

Semenov, Andrey, 2J

Seteikin, A. Y., 2L

Sharma, Y., $2 M$

Shawkey, Heba A., 11

Shevchenko, M. A., 21

Shevchuk, Alisa I., 22

Shkondin, Evgeniy, 24

Smirnov, Aleksei N., 22

Solovyeva, Elena V., 22

Starovoytov, Anton A., 20

Stolt, Timo, $1 \mathrm{~T}$

Strangi, Giuseppe, 24

Strelnikov, Aleksei S., 22

Takayama, Osamu, 24

Taliercio, Thierry, 16

Toropov, Nikita A., 20

Trull, J., IR

Ünalan, H. Emrah, 2F

Vaippully, Rahul, OY

Vesala, Anna, 1T

Vidal, Xavier, 07

Vincent, Grégory, 16

Wall, S., IR

Yaroshenko, Vitaly, 2J, 2P

Zambrana-Puyalto, Xavier, 07

Zang, Xiaorun, $1 \mathrm{~T}$

Zhou, J., 10

Zolotovskii, Igor, 21
Zuev, Dmitry, 2J, 2P

Zyss, J., 10

Zyubin, Andrey Y., 2C, 2l, 2L 


\title{
Conference Committee
}

\author{
Symposium Chairs
}

Francis Berghmans, Vrije Universieit Brussel (Belgium)

Thierry Georges, Oxxius SA (France)

Paul C. Montgomery, Université de Strasbourg (France)

Lluis Torner, ICFO Barcelona (Spain)

Conference Chairs

David L. Andrews, University of East Anglia (United Kingdom)

Angus J. Bain, University College London (United Kingdom)

Martti Kauranen, Tampere University of Technology (Finland)

Jean-Michel Nunzi, Queen's University (Canada)

Conference Program Committee

Antonio Ambrosio, Harvard University (United States)

Sophie Brasselet, Institut Fresnel (France)

Michele Celebrano, Politecnico di Milano (Italy)

Crina M. Cojocaru, Universidad Politècnica de Catalunya (Spain)

Céline Fiorini-Debuisschert, Commissariat à l'Énergie Atomique (France)

Vincent Ginis, Vrije Universiteit Brussel (Belgium)

Arseniy I. Kuznetsov, A*STAR - Institute of Materials Research and Engineering (Singapore)

Christoph Lienau, Carl von Ossietzky Universität Oldenburg (Germany)

Raúl J. Martín-Palma, Universidad Autónoma de Madrid (Spain)

Jesper Mork, Technical University of Denmark (Denmark)

Jean-Luc Pelouard, Centre de Nanosciences et de Nanotechnologies (France)

Mohsen Rahmani, The Australian National University (Australia)

Monika Ritsch-Marte, Medizinische Universität Innsbruck (Austria)

Kalaichelvi Saravanamuttu, McMaster University (Canada)

Haim Suchowski, 3DOptix (Israel)

Jun Wang, Shanghai Institute of Optics and Fine Mechanics, Chinese Academy of Sciences (China)

Anatoly V. Zayats, King's College London (United Kingdom)

Session Chairs

1 Welcome and Opening Presentation

Angus J. Bain, University College London (United Kingdom) 
2 Structured Light and Applications

David L. Andrews, University of East Anglia (United Kingdom)

3 Nanophotonics Structures

Martti Kauranen, Tampere University (Finland)

4 Optical Manipulation

David L. Andrews, University of East Anglia (United Kingdom)

5 Topological Fields

Andrew Forbes, University of the Witwatersrand, Johannesburg (South Africa)

6 Plasmonics 1

Benjamin J. Eggleton, The University of Sydney (Australia)

7 Plasmonics $\|$

Vincent Ginis, Harvard University (United States)

8 Plasmonics and Nanoantennae

Martti Kauranen, Tampere University (Finland)

9 Novel Synthetic Methods

Jean-Michel Nunzi, Queen's University (Canada)

10 Sensing

Arseniy I. Kuznetsov, A*STAR - Data Storage Institute (Singapore)

11 Photoinduced Effects

Raúl J. J. Martín-Palma, Universidad Autónoma de Madrid (Spain)

12 Nanophotonics and Metamaterials: Joint Session

Anatoly V. Zayats, King's College London (United Kingdom)

13 Photonic Sources

Rachel Grange, ETH Zurich (Switzerland)

14 Guided Modes and Cavities

Christoph Lienau, Carl von Ossietzky Universität Oldenburg (Germany)

15 Time Resolved Dynamics

Angus J. Bain, University College London (United Kingdom)

16 Nonlinear Interactions

Jean-Michel Nunzi, Queen's University (Canada) 